\title{
HOMOTOPIC CURVES ON SURFACES
}

\author{
HAROLD I. LEVINE ${ }^{1}$
}

In the following, a surface is always a compact, orientable, twodimensional manifold with or without boundary, a closed surface is one without boundary, and a simple, closed curve on a surface is a closed, connected, one-dimensional submanifold. For simplicity in referring to curves, no notational distinction is made between the embedding of the circle and the image under the embedding - the context will make it clear which is intended.

If $W$ is a surface whose boundary consists of $n$ disjoint, simple, closed curves, the genus of $W, g(W)$, is defined to be the genus of a surface without boundary obtained by attaching a 2-cell to each of the boundary curves. The relation between the genus of $W$ and its inner Euler characteristic, $\chi(W)$ is:

$$
\chi(W)=2-2 g-n .
$$

Any two surfaces with the same number of boundary curves and genus are homeomorphic [2], and if everything is differentiable, diffeomorphic [1]. A surface with one boundary curve and genus zero will be called a disc, and a surface with two boundary curves and genus zero will be called a cylinder.

No distinction is made between the topological and differentiable cases, since everything below applies equally well to both.

The object of this note is to prove:

TheOREM. Let $Q$ and $\&$ be simple, closed, nonintersecting curves on a closed surface, $V$, then:

(i) If $Q$ is homotopic to zero, then a bounds a disc in $V$.

(ii) If $Q$ and $B$ are freely homotopic but not homotopic to zero, then $Q \cup B$ bounds a cylinder in $V$.

The author wishes to thank R. Palais for many helpful conversations.

1. Lemma. Suppose $a_{1}, \cdots, a_{n}$ are simple, closed, nonintersecting, null-homologous curves in $V$, then $V-U a_{i}$ has $(n+1)$ components.

Proof. Consider the cohomology sequence of the pair $(V, A)$, where

Received by the editors September 21, 1962.

${ }^{1}$ This work was supported in part by the National Science Foundation under grant NSF-G14227. 
$A=\bigcup a_{i}:$

$$
\cdots \rightarrow H^{1}(V) \stackrel{i^{*}}{\rightarrow} H^{1}(A) \rightarrow H^{2}(V, A) \rightarrow H^{2}(V) \rightarrow H^{2}(A) \rightarrow \cdots .
$$

Since all of the $a_{i}$ are null-homologous $i^{*}$ is the zero map. $H^{1}(A) \approx Z^{n}$, $H^{2}(A)=0$, and $H^{2}(V) \approx Z$. Thus we have:

$$
0 \rightarrow Z^{n} \rightarrow H^{2}(V, A) \rightarrow Z \rightarrow 0 .
$$

So $H^{2}(V, A) \approx Z^{n+1}$, and the result follows by duality, $H^{2}(V, A)$ $\approx H_{0}(V-A)$.

Lemma. Suppose $a_{1}, \cdots, a_{n}$ are simple, closed nonintersecting, homologous, but not null-homologous curves on $V$, then $V-U a_{i}$ has $n$ components.

Proof. This proof is similar to the preceding one except that now $i^{*}$ maps $H^{1}(V)$ onto the diagonal of $Z^{n}$. The resulting exact sequence is:

$$
0 \rightarrow Z \rightarrow Z^{n} \rightarrow H^{2}(V, A) \rightarrow Z \rightarrow 0,
$$

and so $H^{2}(V, A) \approx H_{0}(V-A) \approx Z^{n}$.

2. The theorem breaks up into three cases: (i) $a$ homotopic to zero, (ii) $)_{1} Q$ and $Q$ freely homotopic, homologous to zero but not homotopic to zero, (ii) $)_{2} Q$ and $B$ freely homotopic but not homologous to zero.

In (i), since $Q$ is homologous to zero the surface is divided by $a$ into two surfaces with genera $g^{\prime}$ and $g^{\prime \prime}$ and the assertion of the theorem is that $g^{\prime}$ or $g^{\prime \prime}$ is zero. Since the genus of the whole surface, $g=g^{\prime}+g^{\prime \prime}$, we see that the result is immediate if $g \leqq 1$.

In (ii) ${ }_{1}$, since the curves $Q$ and $B$ are null-homologous, $V$ is divided by $Q$ and $B$ into three surfaces: two with single bounding curves with genera $g^{\prime}$ and $g^{\prime \prime \prime}$ and one with two boundary curves with genus $g^{\prime \prime}$. Since neither $Q$ nor $B$ is null-homotopic, $g^{\prime}$ and $g^{\prime \prime \prime}$ are nonzero. The assertion of (ii) $)_{1}$ is that $g^{\prime \prime}$ is zero. Since the genus of $V, g=g^{\prime}+g^{\prime \prime}$ $+g^{\prime \prime \prime}$, the conclusion of (ii) ${ }_{1}$ holds for surfaces of genus $g \leqq 2$ if $a$ and $B$ are null-homologous and not null-homotopic (which is only possible if $g=2$ ).

In (ii) ${ }_{2}$ since the curves $Q$ and $B$ are homologous but not nullhomologous, $V$ is divided by $Q$ and $B$ into two surfaces with two boundary curves each and genera $g^{\prime}$ and $g^{\prime \prime}$. The assertion of (ii) is that either $g^{\prime}$ or $g^{\prime \prime}$ vanishes. Since the genus of $V, g=g^{\prime}+g^{\prime \prime}+1$, the conclusion of (ii) ${ }_{2}$ follows if $g \leqq 2$. Thus:

(ii)' If $V$ is a closed surface of genus $g \leqq 2$ and if $Q$ and $B$ are simple, 
closed, nonintersecting curves which are homologous but not homotopic to zero in $V$, then $Q \cup B$ is the boundary of a cylinder in $V$.

3. If we assume (i) of the theorem, then the case of (ii) in which $Q$ and $B$ are homologous to zero follows at once. For suppose $Q$ and $B$ divide $V$ into $V^{\prime}, V^{\prime \prime}$, and $V^{\prime \prime \prime}$, with boundaries $Q, Q \cup B$, and $B$ respectively. Since $a$ is not null-homotopic we know that $g^{\prime} \neq 0$. Construct a new surface, $W$, by attaching a disc, $D$, along $\circledast$ to $V^{\prime} \cup V^{\prime \prime}$. Now $Q$ and $B$ are null-homotopic and therefore $Q$ bounds a disc in $W$. Since $Q$ is the boundary in $W$ of $V^{\prime}$ and $V^{\prime \prime} \cup D$ and since $V^{\prime}$ is not a disc $V^{\prime \prime} \cup D$ must be. Thus $g^{\prime \prime}=g\left(V^{\prime \prime} \cup D\right)=0$, so $V^{\prime \prime}$ is a cylinder.

4. Part (i) of the theorem is an immediate consequence of Van Kampen's Theorem [3]. Let $a$ be a simple, closed, null-homotopic curve in $V$. The curve divides $V$ into two components whose closures are $V^{\prime}$ and $V^{\prime \prime}$ with common boundary, $a$. Let $T$ be an open tubular $n b h d$ about $a$ and let $V_{1}=V^{\prime} \cup T$ and $V_{2}=V^{\prime \prime} \cup T .\left\{V_{1}, V_{2}\right\}$ is an open cover of $V$ and $T=V_{1} \cap V_{2}$ is connected. Let $x_{0} \in a$; we compute $\pi_{1}\left(V, x_{0}\right)$ from $\pi_{1}\left(V_{i}, x_{0}\right)$ and $\pi_{1}\left(T, x_{0}\right)$. Let $\alpha, \alpha_{i}$ be the homotopy class of $Q$ in $\pi_{1}\left(T, x_{0}\right), \pi_{1}\left(V_{i}, x_{0}\right), i=1,2 . \pi_{1}\left(V_{1}, x_{0}\right)$ has generators $\alpha_{1}, A_{i}, B_{i}, i=1, \cdots, g^{\prime}$, with one relation $\alpha_{1}=\left[A_{1}, B_{1}\right] \cdot\left[A_{2}, B_{2}\right] . \cdots$ - $\left[A_{g^{\prime}}, B_{g^{\prime}}\right]=w_{1}$, where $[X, Y]$ is the commutator of $X$ and $Y$ and $g^{\prime}=g\left(V^{\prime}\right)$. Similarly $\pi_{1}\left(V_{2}, x_{0}\right)$ has generators $\alpha_{2}, C_{j}, D_{j}, j=1, \cdots, g^{\prime \prime}$, with one relation $\alpha_{2}=\left[C_{1}, D_{1}\right] \cdot\left[C_{2}, D_{2}\right] \cdots\left[C_{g^{\prime \prime}}, D_{g^{\prime \prime}}\right]=w_{2}$, where $g^{\prime \prime}=g\left(V^{\prime \prime}\right) . \pi_{1}\left(T, x_{0}\right)$ has one generator, $\alpha$, with no relations. Applying Van Kampen's Theorem we have $\pi_{1}\left(V, x_{0}\right)$ is generated by $\alpha, \alpha_{1}$, $\alpha_{2}, A_{i}, B_{i}, C_{j}, D_{j} ; i=1, \cdots, g^{\prime} ; j=1, \cdots, g^{\prime \prime}$ with the relations:

$$
\alpha=\alpha_{1}=\alpha_{2}=w_{1}=w_{2} .
$$

Or $\pi_{1}\left(V, x_{0}\right)$ is generated by $A_{i}, B_{i}, C_{j}, D_{j}$ with the one relation $w_{1}=w_{2}$. We show that the two assumptions $g^{\prime}$ and $g^{\prime \prime}$ nonzero and $Q$ null-homotopic in $V$ are incompatible. In $\pi_{1}\left(V, x_{0}\right)$ the class of $a$ is represented by the images of $w_{1}$ and $w_{2}$. Thus if this class is 1 in $\pi_{1}\left(V, x_{0}\right)$, we must be able to express $w_{1}$ as a word in the conjugates of powers of $w_{1}^{-1} w_{2}$.

Lemma. Let $F$ be the free group with generators $A_{i}, B_{i}, C_{j}, D_{j}$; $i=1, \cdots, g^{\prime}$ and $j=1, \cdots, g^{\prime \prime}$. Let $\left.w_{1}=\left[A_{1}, B_{1}\right] \cdots \cdot A_{g^{\prime}}, B_{\sigma^{\prime}}\right]$ and $w_{2}=\left[C_{1}, D_{1}\right] . \cdots \cdot\left[C_{g^{\prime \prime}}, D_{g^{\prime \prime}}\right]$. If $N$ is the smallest, normal subgroup of $F$ containing $w_{1}^{-1} w_{2}$ then $w_{1} \notin N$.

Proof. Let $H$ be the subgroup of $(3 \times 3)$ real, triangular matrices generated by: 


$$
h_{1}=\left(\begin{array}{lll}
1 & 0 & 0 \\
0 & 1 & 1 \\
0 & 0 & 1
\end{array}\right) \quad \text { and } \quad h_{2}=\left(\begin{array}{lll}
1 & 1 & 0 \\
0 & 1 & 0 \\
0 & 0 & 1
\end{array}\right)
$$

Define two homomorphisms $\psi_{i}: F \rightarrow H$ as follows: $\psi_{1}\left(A_{1}\right)=\psi_{2}\left(C_{1}\right)=h_{1}$, $\psi_{1}\left(B_{1}\right)=\psi_{2}\left(D_{1}\right)=h_{2}$, and $\psi_{2}\left(A_{1}\right)=\psi_{2}\left(B_{1}\right)=\psi_{1}\left(C_{1}\right)=\psi_{1}\left(D_{1}\right)=I ; \psi_{i}$ of any other generator is $I$. Notice that $\psi_{2}\left(w_{1}\right)=I, \psi_{1}\left(w_{1}\right)=\psi_{2}\left(w_{2}\right)=h \neq I$, and that $[H,[H, H]]=I$. If $w_{1} \in N$, then $w_{1}$ is a word in the conjugates of the powers of $w_{1}^{-1} w_{2}$. Thus for some integer $k, \psi_{1}\left(w_{1}\right)=\psi_{1}\left(w_{1}\right)^{-k}$ and $\psi_{2}\left(w_{1}\right)=\psi_{2}\left(w_{2}\right)^{k}$. So we have the contradiction, $h=I$.

5. We now complete the proof of (ii). The curves $Q$ and $B$ are freely homotopic, nonintersecting, simple closed curves in $V$ which are not homologous to zero. Let $V^{\prime}$ and $V^{\prime \prime}$ be the closures of the components of $V-a \cup \beta$. $V^{\prime}$ and $V^{\prime \prime}$ have $Q \cup \beta$ as their common boundary. Let $M$ and $N$ be disjoint tubular $n b h d s$ of $Q$ and $B$, respectively. Let $V_{1}=V^{\prime} \cup M \cup N$ and $V_{2}=V^{\prime \prime} \cup M \cup N ; V_{1} \cap V_{2}=M \cup N$. In order to compute the fundamental group of $V$ we use Weinzweig's generalization of Van Kampen's Theorem [4].

Pick a point $x_{0} \in Q$ and $y_{0} \in ß$. Since both $x_{0}$ and $y_{0}$ are in $V_{1} \cap V_{2}$ we may pick arcs $d_{i}: I \rightarrow V_{i}$ such that $d_{i}(0)=x_{0}$ and $d_{i}(1)=y_{0}$. Let D: $I \rightarrow V$ be defined by:

$$
D(t)= \begin{cases}d_{1}(2 t), & \text { if } 0 \leqq t \leqq 1 / 2, \\ d_{2}(2-2 t), & \text { if } 1 / 2 \leqq t \leqq 1\end{cases}
$$

Let $\alpha_{\imath}$ be the class of $\alpha$ in $\pi_{1}\left(V_{i}, x_{0}\right)$, and $\beta_{i}$ be the class of $d_{i} B d_{i}^{-1}$ in $\pi_{1}\left(V_{i}, x_{0}\right)$. Let $\alpha$ be the class of $Q$ in $\pi_{1}\left(M, x_{0}\right)$ and $\beta$ be the class of $B$ in $\pi_{1}\left(N, y_{0}\right) . \pi_{1}\left(M, x_{0}\right)$ and $\pi_{1}\left(N, y_{0}\right)$ are infinite cyclic with generators $\alpha$ and $\beta$, respectively. $\pi_{1}\left(V_{1}, x_{0}\right)$ has generators $\alpha_{1}, \beta_{1}, A_{i}, B_{i}$; $i=1, \cdots, g^{\prime}=g\left(V^{\prime}\right)$; with the one relation $\alpha_{1}=\beta_{1}\left[A_{1}, B_{1}\right] . \ldots$ - $\left[A_{g^{\prime}}, B_{g^{\prime}}\right]=\beta_{1} w_{1} . \pi_{1}\left(V_{2}, x_{0}\right)$ has generators $\alpha_{2}, \beta_{2}, C_{j}, D_{j} ; j=1, \cdots, g^{\prime \prime}$ $=g\left(V^{\prime \prime}\right) ;$ with the one relation $\alpha_{2}=\beta_{2}\left[C_{1}, D_{1}\right] \cdots \cdot\left[C_{g^{\prime \prime}}, D_{g^{\prime \prime}}\right]$ $=\beta_{2} w_{2}$. Using Theorem 2 of [4], we see that $\pi_{1}\left(V, x_{0}\right)$ is generated by: $\alpha, \alpha_{1}, \alpha_{2}, \beta, \beta_{1}, \beta_{2}, A_{i}, B_{i}, C_{j}, D_{j}$, and $\delta$, the class of $D$. The relations are: $\alpha=\alpha_{1}=\alpha_{2}, \beta=\beta_{1}=\delta \beta_{2} \delta^{-1}, \alpha_{1}=\beta_{1} \cdot w_{1}$, and $\alpha_{2}=\beta_{2} \cdot w_{2}$. Or $\pi_{1}\left(V, x_{0}\right)$ is generated by: $\beta, \delta, A_{i}, B_{i}, C_{j}, D_{j}$ with the relation $\beta w_{2}=\delta \beta \delta^{-1} w_{1}$.

Again we show that the assumptions that $Q$ and $B$ are freely homotopic and that $g^{\prime}$ and $g^{\prime \prime}$ are nonzero are incompatible. If we let $G$ be the quotient group obtained from $\pi_{1}\left(V, x_{0}\right)$ by introducing the relations $\beta=1$ and $\delta=1, G$ is the group generated by $A_{i}, B_{i}, C_{j}, D_{j}$ with the relation $w_{1}=w_{2}$. If $Q$ and $B$ are freely homotopic then the image 
of $w_{1}$ in $G$ is 1 , but by the lemma of the preceding section this is possible only when either $g^{\prime}$ or $g^{\prime \prime}$ is zero.

\section{REFERENCES}

1. J. Munkres, Obstructions to the smoothing of piecewise differentiable homeomorphisms, Ann. of Math. (2) 72 (1960), 521-554.

2. H. Seifert and W. Threlfall, Lehrbuch der Topologie, Teubner, Leipzig, 1934.

3. E. Van Kampen $O n$ the connection between fundamental groups of some related spaces, Amer. J. Math. 55 (1933), 261-267.

4. A. I. Weinzweig, The fundamental group of a union of spaces, Pacific J. Math. 11 (1961), 763-776.

BrandeIs UnIVERSITY 